\title{
A Front Tracking Method for Conservation Laws in One Dimension ${ }^{1}$
}

\author{
N. H. RISEBRo* \\ A. TVEITo ${ }^{* *}$ \\ *Department of Mathematics \\ University of Oslo \\ P.O. Box 1053, Blindern \\ $\mathrm{N}-0316$ Oslo 3, Norway \\ ** Department of Informatics \\ University of Oslo \\ P.O. Box 1080, Blindern \\ N-0316 Oslo 3, Norway
}

\begin{abstract}
We present a front tracking technique for conservation laws in one dimension. The method is based on approximations to the solution of Riemann problems where the solution is represented by piecewise constant states separated by discontinuities. The discontinuities are tracked until they interact, at this point a new Riemann problem is solved and so on. This method is tested on the system of nonstationary gas dynamics defined by the Euler equations. Three test cases are presented and the front tracking method is compared with the random choice method and with the Lax-Friedrichs method.
\end{abstract}

1. Introduction. Over the last 40 years many numerical methods have been developed to facilitate the analysis of nonstationary gasdynamics. A major difficulty in this area of numerical modelling is the presence of shocks and contact discontinuities in the solution. To represent these satisfactorily the various finite difference methods demand such a large number of grid nodes and time steps, that accurate simulation of compressible gas flow with these methods represents enormous computer tasks.

In order to overcome the difficulty of representing discontinuities in the flow, we have two main approaches at our disposal: The random choice method and front tracking/capturing methods. The random choice method, also known as Glimm's method, was originally described by Glimm [7] as a theoretical tool to obtain existence results for one dimensional strictly hyperbolic conservation laws with small initial data, but was later developed into a practical computational tool by Chorin[3], cf. also [4],[20],[10]. Glimm's method has been shown to be an accurate tool $[4],[20],[6]$, but it involves the solution of Riemann problems at each point in the numerical grid, even in the smooth regions of the flow. Hence the method is relatively time consuming compared to the simpler difference methods.

Since the number of large discontinuities in many applications is small, front tracking has been proposed as an accurate and efficient method to deal with the problem of discontinuities. Front tracking is not one method, but a collective name used for many

\footnotetext{
${ }^{1}$ This work was supported in part by the Royal Norwegian Council for Technical and Industrial Research and by VISTA, a reasearch cooperation between the Norwegian Academy of Science and Letters and Den norske stats oljeselskap a.s. (Statoil)
} 
methods which use some kind of special treatment for steep gradients or discontinuities in the flow. Some of these methods track large discontinuities and use standard methods in the smoother parts of the solution. This approach has been used successfully in some one-[2] and two-dimensional studies [9], and in the simulation of fluid flow in oil reservoirs [8].

Another approach to front tracking is motivated by Dafermos'[5] method for the scalar conservation law in one space dimension. Dafermos approximated the flux function by a piecewise linear function. The advantage of this is that the solution of the Riemann problem is piecewise constant, and one can therefore solve the Cauchy problem with piecewise constant initial data exactly for all time. Subsequent work on Dafermos' method with a scalar conservation law was done by LeVeque[16], Lucier[17] and Holden et al[14]. There has been some work on generalizations of Dafermos' method to systems of conservation laws; Hedstr $\varnothing \mathrm{m}[12]$ did some numerical experiments on the $p$-system for a piecewise linear function $p(u)$. We will here present another front tracking scheme inspired by Dafermos' method. In [18] it was proposed to approximate the solution of the Riemann problem by a step function without modifying the flux functions. Roughly speaking this step function should have large discontinuities where the correct solution to the Riemann problem has discontinuities, and have "small steps" in the smooth part of the solution. The discontinuities in the approximated solution are assigned a speed which is the correct speed of the discontinuity if it represents a discontinuity in the correct solution, or else it has the characteristic speed of the state immediately to the left of the discontinuity. These discontinuities are then tracked until two or more collide. At the collision point we solve a Riemann problem with left and right states given by the states to the left and right of the collision. The solution of this Riemann problem is approximated in the same way and the tracking is continued untion the next collision and so on.

In section two we give a description of this front tracking method in general, and in section three we explain how it is used to simulate the compressible nonstationary fluid flow given by the Euler equations. In section four of this study we present three test cases for the front tracking method: First a closed tube problem proposed by Woodward in $[\mathbf{2 1}],[\mathbf{2 2}]$, secondly a problem where the front tracking method is compared with the LaxFriedrichs method and with the Random choice method. The second problem is chosen so that there will be many interactions of tracked discontinuities, and this seems to be a difficult problem for front tracking. The front tracking method shows great promise with respect to the study of asymptotic behavior for large time, therefore we include a third test example where the initial function is an "almost Riemann problem", i.e. a single smooth but steep transition between constant states.

2. The front tracking method. The general front tracking method we present here is a generalization of Dafermos' method for scalar conservation laws. In principle the method is applicable whenever a solution of the Riemannn problem is computable, although we have no proof of convergence for arbitrary initial data. But for strictly hyperbolic systems with sufficiently small total variation of the initial data, it was proved in [18] that the method produces a subsequence of approximate solutions which converges towards a weak solution of the problem. More precisely, [18] gives an alternative proof of Glimm's famous existence theorem[7]. The purpose of the present paper is to explore the method as a 
computational tool.

Consider the following system of conservation laws

$$
u_{t}+f(u)_{x}=0
$$

where $u=u(x, t) \in \mathbb{R}^{n}$ and $f: \mathbb{R}^{n} \rightarrow \mathbb{R}^{n}$ is a smooth function. The Riemann problem for (2.1) is the initial value problem with data of the form

$$
u(x, 0)=\left\{\begin{array}{l}
u_{l} \text { if } x<0 \\
u_{r} \text { if } x>0
\end{array}\right.
$$

If $u(x, t)$ is a solution of $(2.1),(2.2)$ then so is $u(c x, c t)$ for all constants $c>0$, therefore to be unique the solution of a Riemann problem has to be self similar i.e. $u(x, t)=\bar{u}(x / t)$. The solution of the Riemann problem is composed of elementary waves, i.e. rarefaction waves and shock waves (including contact discontinuities), and constant states.

Rarefaction waves are classical solutions of the system (2.1). For such solutions (2.1) can be rewritten as

$$
u_{t}+d f(u) u_{x}=0
$$

where $d f$ is the Jacobian matrix of $f$. For self similar solutions we get

$$
(d f-(x / t) I) \bar{u}(x / t)=0 .
$$

Hence $x / t$ is an eigenvalue of $d f$ and $\bar{u}(x / t)$ is the corresponding eigenvector. Let $\lambda$ be an eigenvalue of $d f$ and let $r$ be the corresponding right eigenvector. If $\bar{u}$ is an integral curve of $r$, i.e.

$$
\bar{u}^{\prime}(\xi)=r(\bar{u}(\xi))
$$

and $\bar{u}$ connects the states $u_{l}$ and $u_{r}$ and if $\lambda$ increases as $\bar{u}$ goes from $u_{l}$ to $u_{r}$, then the path $\bar{u}$ traverses is called a rarefaction curve. Rarefaction curves are directed towards increasing values of $\lambda$, and the function

$$
u(x, t)=\left\{\begin{array}{l}
u_{l} \text { for } x / t<\lambda\left(u_{l}\right) \\
\bar{u}(x / t) \text { for } x / t=\lambda(\bar{u}) \\
u_{r} \text { for } x / t>\lambda\left(u_{r}\right)
\end{array}\right.
$$

is called a simple rarefaction solution of (2.1). The speed of a rarefaction wave is given by $\lambda(\bar{u})$, in particular the initial speed is $\lambda\left(u_{l}\right)$ and the final speed $\lambda\left(u_{r}\right)$.

Shock waves are weak solutions of $(2.1)$ of the form

$$
u(x, t)= \begin{cases}u_{l} & \text { for } x / t<\sigma \\ u_{r} & \text { for } x / t>\sigma\end{cases}
$$


where $\sigma$ is the shock speed. In order to be a weak solution, $\sigma$ has to satisfy the RankineHugoniot relation

$$
\sigma\left(u_{l}-u_{r}\right)=f\left(u_{l}\right)-f\left(u_{r}\right)
$$

A weak solution is not necesarily unique, therefore an additional entropy condition is imposed on (2.8) in order to obtain uniqueness.

The solution of a Riemann problem consists of a sequence of elementary waves connecting the left state $u_{l}$ with the right state $u_{r}$. We introduce the following notation: $u_{1} \stackrel{w}{\rightarrow} u_{2}$ means that the state $u_{1}$ can be connected to $u_{2}$ by an elementary wave $w$. Let $u_{1} \stackrel{w_{1}}{\longrightarrow}$ $u_{2} \stackrel{w_{2}}{\longrightarrow} u_{3}$ be two elementary waves, and let $v_{i}^{I}$ and $v_{i}^{F}$ be the initial and final speed of each wave. If $w_{i}$ is a shock wave then $v_{i}^{I}=v_{i}^{F}=\sigma$. The two waves are said to be compatible if

$$
v_{1}^{F} \leq v_{2}^{I}
$$

If both waves are shocks we impose strict inequality in (2.9). The notation $u_{1} \stackrel{w_{1}}{\longrightarrow} u_{2} \stackrel{w_{2}}{\longrightarrow}$ $u_{3}$ means that $w_{1}$ and $w_{2}$ are compatible, and that $u_{1}$ can be connected to $u_{3}$ through the intermediate state $u_{2}$. A solution of a Riemann problem thus consists of a sequence of compatible waves

$$
u_{l}=u_{0} \stackrel{w_{1}}{\longrightarrow} u_{2} \stackrel{w_{2}}{\longrightarrow} \cdots \stackrel{w_{N}}{\longrightarrow} u_{r} .
$$

Such a solution can be computed explicitly for many systems of equations, both for strictly hyperbolic systems[11],[19], and for systems of non strictly hyperbolc[15] or mixed type[13].

Assume that the solution of the Riemann problem (2.2) for the system (2.1) can be represented on the form (2.10). We shall construct a piecewise constant approximation $u_{\delta}(\xi)$ to the solution, represented by the sequences $\left\{u_{\delta, j}\right\},\left\{\xi_{\delta, j}\right\}$ in the following manner

$$
u_{\delta}(\xi)=u_{\delta, i} \quad \text { for } \quad \xi_{\delta, i}<\xi<\xi_{\delta, i+1} .
$$

Let $\left\{u_{i}, w_{i}, v_{i}^{I}, v_{i}^{F}\right\}_{i=0}^{N}$ denote the solution of the Riemann problem given by (2.10). We define $\left\{u_{\delta, j}\right\}$ and $\xi_{\delta, j}$ recursivly as follows

$$
u_{\delta, 0}=u_{0}=u_{l} \quad \xi_{\delta, 0}=-\infty
$$

Assume we have defined the sequences $\left\{u_{\delta, j}\right\}_{j=0}^{k}$, and $\left\{\xi_{\delta, j}\right\}_{j=0}^{k}$ and that $u_{\delta, k}=u_{i}$ for some $i$. The approximation of the wave $u_{i} \stackrel{w_{i+1}}{\longrightarrow} u_{i+1}$ can be divided into two cases:

(1) $w_{i}$ is a shock wave. In this case $\xi_{\delta, k+1}=u_{i}$ and $u_{\delta, k+1}=u_{i+1}$.

(2) $w_{i}$ is a rarefaction wave. Let

$$
\begin{aligned}
& M=\left\lceil\frac{\lambda\left(u_{i+1}\right)-\lambda\left(u_{i}\right)}{\delta}\right]+1 \\
& \delta^{\prime}=\frac{\lambda\left(u_{i+1}\right)-\lambda\left(u_{i}\right)}{M}
\end{aligned}
$$


where $\lceil\cdot\rceil$ is the truncation operator, and let $\bar{u}$ be the rarefaction curve defined by (2.5). We can assume $\bar{u}$ to be parametrisized by $\lambda$. Now let

$$
u_{\delta, k+l}=\bar{u}\left(\lambda\left(u_{i}\right)+l \delta^{\prime}\right)
$$

and

$$
\xi_{\delta, k+l}=\lambda\left(u_{\delta, k+l}\right)
$$

for $l=1, \ldots, M$, cf. figure 2.1 and 2.2 .

We now say that (2.11) is a $\delta$-approximation to the solution of the Riemann problem. This approximation has the properties that

$$
\begin{array}{r}
\left\{u_{i}\right\} \subset\left\{u_{\delta, j}\right\} \\
\sup _{\xi}\left|u_{\delta}(\xi)-u(\xi)\right|=O(\delta) .
\end{array}
$$

Observe that by (2.16) all intermediate states in the solution of the Riemann problem are kept in the approximation. This is due to the separate treatment of each elementary wave. We believe that this property of the approximation is crucial in order for it to be able to reflect the complexity of the solution.

This $\delta$-approximation to the solution of the Riemann problem is the building block of our front tracking scheme. The $\delta$-approximation defines of a number of fronts. A front is an object with the following attributes

$$
\text { front : }\left\{u_{l}, s,\left(x_{0}, t_{0}\right),\left(x_{1}, t_{1}\right), \text { family, pointers }\right\} \text {. }
$$

Here $u_{l}$ is the state to the left of the discontinuity, $s$ is the speed of the discontinuity, $\left(x_{0}, t_{0}\right)$ is the point in $x, t$ space where the discontinuity originates, and $\left(x_{1}, t_{1}\right)$ is the point it terminates. A front is terminated when it collides with one of its neighboring fronts. The family of a front indicates the wave type it approximates. For strictly hyperbolic systems it is defined to be $i$ if $v_{i-1}^{F} \leq s<v_{i+1}^{I}$. The pointers are used to store the fronts in a suitable data structure. The fronts are organized from left to right in a so called $x$-list. Each front has a pointer to its left and right neighbor. This structure makes it easy to remove and add fronts. We also have a $t$-list. This list organizes the fronts with respect to the collision times $t_{1}$. The first front in the list has the smallest value of $t_{1}$. This front has a pointer to the front with the second smallest $t_{1}$ and so on.

We are interested in the Cauchy problem for (2.1) with

$$
u(x, 0)=u_{0}(x) .
$$

If $u_{0}$ is a piecewise constant function with a finite number of discontinuities, we can construct a $\delta$-approximation to the solution of each of the Riemann problems defined at the discontinuities of $u_{0}$. We now have a system of fronts, each of which can be propagated independently until one of them interact (collide) with one (or more) of its neighbors. Then we solve the Riemann problem defined by the left state of the left colliding front and the right state of the right colliding front. We make a $\delta$-approximation to this solution, and again we have a system of fronts which can be tracked until the next collision. In this way the solution can be advanced in time, see figure 2.3. Now we can state the algorithm used to compute our numerical approximation: 


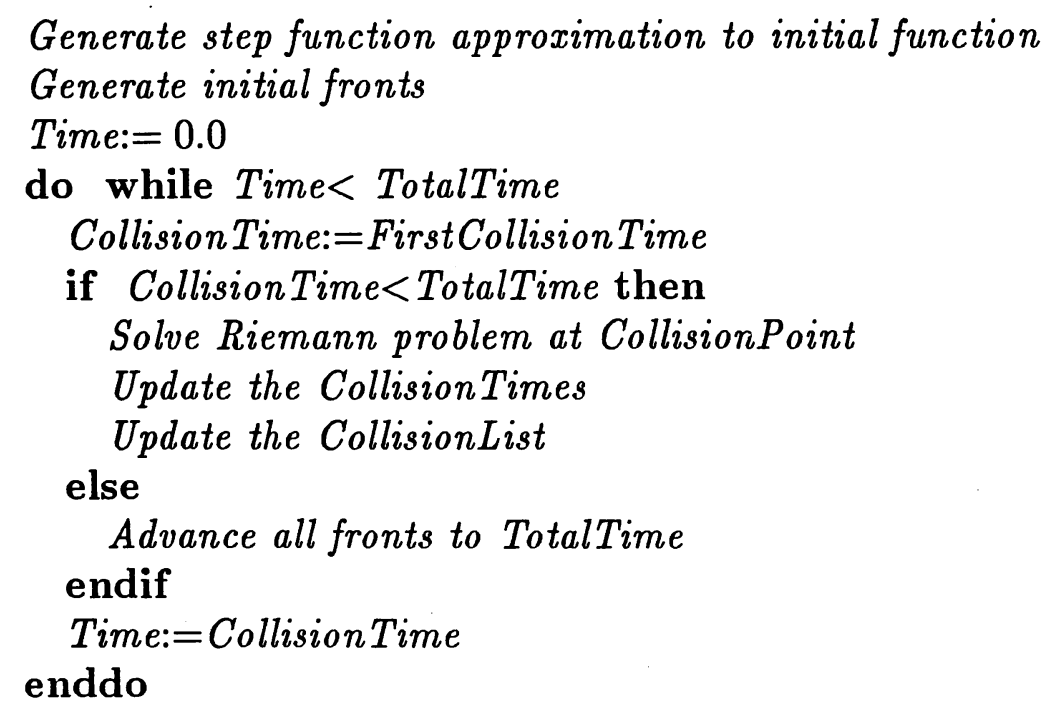

This front tracking method is fast compared to fixed grid methods since it automatically will focus computational effort where interactions are occurring, i.e. where the solution has a complex behavior. This effect is espescially apparent when few interactions are taking place as we show in example 3 in section four.

Remark. When updating the solution in this way, we in general have no theoretical limit on the number of fronts that may arise. Therefore we must have some mechanism which limits the growth of the number of fronts. In order to do this, we have chosen to ignore small fronts in the $\delta$-approximation to the solution of the Riemann problem. We ignore fronts which represent a discontinuity of magnitude less than some $c(\delta)$, where $c \rightarrow 0$ as $\delta \rightarrow 0$. In general we have no justification for ignoring such fronts, but for the system of gas-dynamics the numerical experiments showed that this resulted in an accurate and efficient method. In the case of a strictly hyperbolic system with initial data with small total variation, all waves will be weak and Glimm's[7] interaction estimate

$$
e_{k}=\delta_{k i} d_{i}+\delta_{k j} d_{j}+O\left(\left|d_{i}\right|\left|d_{j}\right|\right)
$$

applies. Here $d_{i}, d_{j}$ is the strength of the colliding fronts of family $i$ and $j$ respectively, $e_{k}$ is the strength of the emerging waves of family $k$, and $\delta_{i j}$ is the Kronecker delta. This estimate says that the emerging fronts of families different from the ones colliding will have small strenght if the strengths of the colliding fronts are small.

3. Application to gas-dynamics. We shall consider the equations of one-dimensional gas-dynamics in Eulerian coordinates:

$$
\begin{aligned}
\rho_{t}+(\rho u)_{x} & =0 \\
(\rho u)_{t}+\left(p+\rho u^{2}\right)_{x} & =0 \\
{\left[\rho\left(\frac{1}{2} u^{2}+e\right)\right]_{t}+\left[\rho u\left(\frac{1}{2} u^{2}+e\right)+p u\right]_{x} } & =0 .
\end{aligned}
$$

Here $p$ is the pressure, $u$ is velocity, $\rho$ is the density and $e$ is the internal energy. We assume the gas to be polytropic so $e=c_{v} T$ where $c_{v}$ is a positive constant and $T$ is the 
temperature. We also assume the gas to be ideal so that $p=R \rho T$ where $R$ is a positive constant. Finally we assume that some $\gamma$-gas law holds so $p=k \exp \left(S / c_{v}\right) \rho^{\gamma}$, where $S$ is the entropy and $k$ a positive constant. Since a solution to (3.1) may contain discontinuities, we interpret (3.1) in the sense of distributions.

An integral component of the front tracking method is the solution of the Riemann problem. For the system of gas-dynamics, this solution is well known and has been discussed extensively, see e.g.[19, and the references therein] and [11] where detailed instructions for constructing a solution is given, as well as a comparison between several solution methods. Therefore we will only outline the structure of the solution. The Riemann problem is the special initial value problem for (3.1) where the initial functions take the following form:

$$
(p, u, \rho)(x, 0)= \begin{cases}\left(p_{l}, u_{l}, \rho_{l}\right) & \text { for } x<0 \\ \left(p_{r}, u_{r}, \rho_{r}\right) & \text { for } x>0 .\end{cases}
$$

There are three elementary waves in the solution of the Riemann problem: shocks, rarefaction waves and contact discontinuities. The solution generally contains a leftward moving shock or rarefaction wave, a contact discontinuity, and a rightward moving shock or rarefaction. In fact, given $\left(p_{l}, u_{l}, \rho_{l}\right)$ and $\left(p_{r}, u_{r}, \rho_{r}\right)$ we can construct two curves in the $(p, u)$ plane, $u_{1}$ and $u_{2}$, passing through $\left(p_{l}, u_{l}\right)$, which have the property that if $\left(p_{r}, u_{r}\right)$ is on the curve $u_{1}$, the Riemann problem is solved by a leftward moving wave and possibly a contact discontinuity. If $\left(p_{r}, u_{r}\right)$ is on the curve $u_{2}$ the Riemann problem is solved by a rightward moving wave and possibly a contact discontinuity. Furthermore if $u_{l}>u_{r}$ the wave is a shock wave, and if $u_{l}<u_{r}$ the wave is a rarefaction wave. These curves divide the $(p, u)$ plane into four regions, and the pattern of the solution (e.g. shock-contact-rarefaction) only depends upon which region $\left(p_{r}, u_{r}\right)$ is in, cf. figure 3.1. Similarly there are curves $\tilde{u}_{1}$ and $\tilde{u}_{2}$ through $\left(p_{r}, u_{r}\right)$. The Riemann problem is solved by finding the unique point $\left(p_{m}, u_{m}\right)$ where $\tilde{u}_{2}$ intersects $u_{1}$. The pressure and velocity do not change across the contact discontinuity and the density can be determined when $\left(p_{m}, u_{m}\right)$ is known. Let $\rho_{m} l$ and $\rho_{m r}$ be the densities to the left and right of the contact discontinuity respectively. If the backward moving wave is a shock (rarefaction) we say that $\left(p_{l}, u_{l}, \rho_{l}\right)$ is connected to $\left(p_{m}, u_{m}, \rho_{m l}\right)$ by a shock (rarefaction). Similarly for the forward moving wave. For our purposes we can regard the solution of the Riemann problem to be known with infinite accuracy, although the determination of the middle state usually requires some iterative procedure.

Thus given the solution of the Riemann problem we will, following the general algorithm outlined above, make an approximation to use as a building block in our numerical algorithm. Let $\delta$ be some small positive number. If $\left(p_{i}, u_{i}, \rho_{i}\right)$ is connected to $\left(p_{m}, u_{m}, \rho_{m i}\right)$ by a shock, we leave the discontinuity as it is. If the states are connected by a rarefaction wave we generate constant states on the curve $u_{i}$ (or $\tilde{u}_{i}$ ) in $(p, u, \rho)$ space as in the previous section, cf. (2.13), (2.14) and (2.15). These constant states are generated as follows. First assume that the state to the left is $\left(p_{l}, u_{l}, \rho_{l}\right)$, this will be our first constant state. We then follow the curve $u_{1}$ towards $\left(p_{m}, u_{m}, \rho_{m l}\right)$ a distance $\delta$. This point on the curve will be our next constant state. The speed of the discontinuity between these two constant states will be the characteristic speed to the left of the discontinuity: $u-c$, where $c$ is the sound speed given by $c^{2}=\gamma p / \rho$. We continue with steps of size $\delta$ on the curve $u_{1}$ until we are 
closer (measured in arc-length along the curve) to $\left(p_{m}, u_{m}, \rho_{m l}\right)$ than $\delta,\left(p_{m}, u_{m}, \rho_{m l}\right)$ is then the last constant state. If the state on the left is $\left(p_{m}, u_{m}, \rho_{m r}\right)$ the wave is a forward moving rarefaction wave. In this case we start from $\left(p_{r}, u_{r}, \rho_{r}\right)$ and choose our constant states on $\tilde{u}_{2}$. Otherwise the procedure is symmetrical. We leave the contact discontinuity in the middle as it is. This approximation we call a $\delta$-approximation to the solution of the Riemann problem.

As in the previous section, a $\delta$-approximation consists of a number of fronts. Each front has a state; $(p, u, \rho)$ which is the constant state immediately to the left of it, a speed, and a family. The family of a front is 1 if the left and right states of the front lie on a $u_{1}$ curve, similarly the family is 2 if the states lie on a $\tilde{u}_{2}$ curve. The family of a contact discontinuity is 0 .

Our strategy is now to approximate the initial data as piecewise constant, solve the Riemann problems at $t=0$, then make $\delta$-approximations to the solutions of the initial Riemann problems, and finally to track each front until it interacts with one of its neighboring fronts. Here we can solve a new Riemann problem with states defined by the states of the colliding fronts, make a $\delta$-approximation to this solution and track the fronts until the next collision. In this way we update the solution in time. In order to do this we equip our fronts with some more structure. A front has a starting point $(x, t)$, as well as a collision time $t_{1}$ which is the time it will collide with one of its neighbors. If the front will not collide this parameter is set to be $\infty$. To order these collision times each front also has a pointer to the front which has the first collision time after the fronts own collision time. Finally each front has a strength which is the distance along the $u_{i}$ curve between the state to the left of the front and the state of the right of the front.

The step function approximation to the initial data is also related to $\delta$. We assume our initial data to be piecewise continuous and constant outside some bounded interval. Let $U=(p, u, \rho)$, and let the initial function take the value $U_{l}$ to the left of this interval and the value $U_{r}$ to the right of it. Fix $x_{0}=-\infty, U_{0}=U_{l}$, and let the sequences $\left\{U_{i}\right\}_{1}^{N}$ and $\left\{x_{i}\right\}_{1}^{N}$ be defined by

$$
x_{i}=\inf _{x>x_{i-1}}\left\{\left|U_{0}(x)-U_{i-1}\right| \geq \delta\right\} \quad U_{i}=U_{0}\left(x_{i}\right)
$$

Redefine $U_{N}=U_{r}$. A collision is said to have a strength equal to the product of the strengths of the colliding fronts. When creating a $\delta$-approximation to the solution of a Riemann problem after a collision of fronts, we will not create new fronts of families different from the families of those that collides if the collision strength is less than $\min \left(\delta / 2, \delta^{2}\right)$.

Most of the CPU-time (more than 95\%) used by the front tracking method is spent solving Riemann problems. It is therefore important to have fast Riemann solvers available. We have used the Riemann solver described and recommended by Gottlieb and Groth in [11].

4. Examples. In this section we present three test examples where the front tracking method has been used. We want to explore some of the properties of the front tracking method, such as the convergence rate and conservation of mass end energy, and to compare it to other numerical methods. 
In case of a strictly hyperbolic conservation law with initial data of small total variation, the front tracking method produces a sequence which converges in $L_{1}$-norm[18]. Hence we use the relative difference in $L_{1}$ between a reference solution and the approximate solution as as measure of the error. Let $\|f\|_{1}=\int|f| d x$ and define the error

$$
\epsilon=\frac{\left\|p_{\delta}-p\right\|_{1}}{\|p\|_{1}}+\frac{\left\|u_{\delta}-u\right\|_{1}}{\|u\|_{1}}+\frac{\left\|c_{\delta}-c\right\|_{1}}{\|c\|_{1}}
$$

Here $c$ is the sound speed. The quantities with subscript $\delta$ are the approximate solutions. Example 1 This example is taken from Woodward and Colella[21] [22], and seems to be a standard test problem in numerical gas-dynamics. The initial condition consists of three constant states of a $\gamma$ gas law, $\gamma=1.4$. The gas is initially at rest between closed walls at $x=0$ and $x=1$. The density is everywhere unity, but the pressure varies:

$$
p(0)= \begin{cases}1000.0 & \text { for } x<0.1 \\ 0.01 & \text { for } 0.1<x<0.9 \\ 100.0 & \text { for } x>0.9\end{cases}
$$

Initially two shock waves develop and interact, while two rarefactions develop and are reflected off the walls at each end of the tube. These reflections again interact with each of the shock waves and a very complicated pattern is quickly established. (See also [22] for a qualitative description of the solution.) In figure 4.1 we see all fronts for an approximation using $\delta=100.0$ from $t=0.0$ to $t=0.038$.

The reference solution to this problem used $\delta=0.8$. At $t=0.038$ approximately 5000 000 collisions of fronts have occurred. The reference solution seems to agree well with the solution reported in [22], both the peaks and the position of the discontinuities all agree. In figure 4.2 we see the reference solution and an approximation using $\delta=20.0$. Note that the positions of all major shocks are virtually identical in the two solutions. In table 1 we show $\delta$, the $L_{1}$ error, the error in mass conservation, the error in energy conservation, and the CPU-time used by the front tracker for this problem. Note that although the front tracker is not conservative, the errors in the mass balance and the energy balance remain small and correlates well with the $L_{1}$ error. Based on standard regression analysis we have calculated exponents $r_{1}$ and $r_{2}$ such that $\epsilon=O\left(\delta^{r_{2}}\right)$ and CPU-time $=O\left(\epsilon^{r_{2}}\right)$. These are given at the bottom of table 1 .

Example 2. This example was constructed in order to compare the front tracking method with two other methods; Glimm's method and Lax-Friedrichs' method. Since the front tracking method will be least efficient when there are many interactions, we constructed an initial value problem which gives many interactions of fronts in a short time. For the Lax-Friedrichs method and the random choice method we used a uniform grid for the space variable, and calculated the optimal time step at each time level according to the CFL condition. For a description of Glimm's method we refer the reader to Chorin[3], or Gottlieb[10].

The problem consisted of an open shock tube with initial velocity everywhere zero, the 
initial sound speed everywhere 10.0, and the pressure distribution was given by:

$$
p_{0}(x)= \begin{cases}f(-7) & \text { for } x<-7 \\ f(x) & \text { for }-7<x<7 \\ f(7) & \text { for } x>7\end{cases}
$$

where

$$
f(x)=11+20 e^{-5 x^{2}}+15 e^{-5(x-3)^{2}} .
$$

See figure 4.4. Also for this problem we used a $\gamma$-gas law with $\gamma=1.4$. We calculated this solution up to $t=0.25$. As a reference solution we used the front tracking method with $\delta=0.03$. This run had more than 9000000 collisions of fronts. In figure 4.6 we see the density of the approximate solutions of the three methods as well as the density of the reference solution, at $t=0.25$. We see that a leftward and a rightward moving wavegroup have formed, and that the solution contains four strong shocks. Both Glimm's method and the front tracker have roughly the right discontinuities in the right places, but the front tracker only uses a fraction of the CPU-time of Glimm's method. The front tracker does not have the same peak values as the reference solution, this is due to the different peak values initially. We see that the Lax-Friedrichs method is reasonably fast, but has poor resolution of the shocks. In table 2 we present CPU-times and errors for the three methods. To invesigate the "convergence rates" of the different methods, we used regression analysis to calculate exponents $r$ such that CPU-time $=O\left(\epsilon^{r}\right)$ for each method. These indicate that the three methods have roughly the same $r$ 's, but note that in order to obtain an error of e.g. 0.06 the front tracking method needs a CPU-time of 21 while the Lax-Friedrichs method needs 622 and Glimm's method does not reach this level of accuracy despite a CPU-time of 700.

Example 3. The front tracking method is well suited to study asymptotic behaviour. In order to investigate this we have chosen the third example such that the initial data are "close to" a Riemann problem. The initial sound speed and velocity are 10.0 and 0.0 respectively, and the initial pressure has the distribution

$$
p_{0}(x)= \begin{cases}210 & \text { for } x<-25 \\ 100(1-\tanh (x))+10 & \text { for }-25<x<40 \\ 10 & \text { for } x>40,\end{cases}
$$

cf. figure 4.5. We calculated the solution to this problem up to $t=2.0$. At this time the solution resembles the solution to the Riemann problem with $p_{l}=210.0$ and $p_{r}=10.0$, exept that the contact discontinutity has not yet developed fully. For this problem the front tracking method was compared to the Lax-Friedrichs method. The reference solution for this problem used $\delta=0.5$. Figure 4.6 shows the solution computed by Lax-Friedrichs method and the solution computed by the front tracker, as well as the reference solution, all at $t=2.0$. Although the solution obtained by the front tracker used less than one tenth of the CPU-time of that obtained by Lax-Friedrichs method, the two solutions have roughly the same accuracy. In table 3 we give the CPU-times and errors for the two methods. The results here are all in the same vein as the result in figure 4.8. 
5. Conclusion. We have described a general front tracking algorithm for conservation laws in one space variable. This algorithm can be used for any system for which the solution to the Riemann problem is computable.

The front tracker was tested on the system of compressible non-stationary gas dynamics. In our test examples the front tracking method gives more accurate solutions in less CPUtime than both the Lax-Friedrichs method and Glimm's method. The front tracker is well suited to study the asymptotic behaviour of an initial value problem. The method is nonconservative, but the errors in the conserved variables are small.

We aim to continue our study of this front tracking algorithm by applying it to a system of equations where the solution of the Riemann problem is more complicated.

\section{REFERENCES}

1. F. Bratvedt, K. Bratvedt, C.F. Buchholz, L. Holden, H. Holden, N.H. Risebro, A new front tracking method for reservoir simulation, SPE-paper \# 19805, (1989).

2. P. Charrier, B. Tessieras, On front tracking methods applied to hyperbolic systems of nonlinear conservation laws, SIAM J. Numer. Anal. 23 (1986), 461-472.

3. A.J. Chorin, Random Choice Solution of Hyperbolic Systems, J. Comp. Phys. 22 (1976), 517-533.

4. P. Colella, Glimm's method for gas dynamics, SIAM J. Sci. Stat. Comput. 3 (1982), 76-110.

5. C.M. Dafermos, Polygonal approximation of solution to the initial value problem for a conservation law, J. Math. Anal. Appl. 38 (1972), 33-41.

6. I.I. Glass, T. Saito, Application of random choice method to problems in gasdynamics, Prog. Aerospace Sci. 21 (1984), 201-247.

7. J. Glimm, Solutions in the large for nonlinear hyperbolic systems of equations, Comm. Pure Appl. Math. 18 (1965), 697-715.

8. J. Glimm, J. Grove, B. Lindquist, O. McBryan, G. Trygvason, The bifurcation of tracked scalar waves, SIAM J. Sci. Stat. Comput. 9 (1988), 61-80.

9. J. Glimm, C. Klingenberg, O. McBryan, B. Plohr, D. Sharp, S. Yaniv, Front tracking and two dimensional Riemann problems, Adv. in Appl. Math. 6 (1985), 259-290.

10. J.J. Gottlieb, Staggered and nonstaggered grids with variable node spacing and local time stepping, J. Comput. Phys. (to appear).

11. J.J. Gottlieb, C.P.T. Groth, Assesment of Riemann solvers for unsteady one dimensional inviscid flow of perfect gases, J. Comput. Phys. 78 (1988), 437-458.

12. G.W. Hedstrom, Some numerical experiments with Dafermos' method for nonlinear hyperbolic equations, in "Lecture notes in mathematics," Springer Verlag, Berlin, 1972, pp. 117-138.

13. H. Holden, On the Riemann problem for a prototype of a mixed type conservation law, Comm. Pure and Appl. Math. 40 (1987), 229-264.

14. H. Holden, L. Holden, R. Høegh-Krohn, A numerical method for first order nonlinear scalar conservation laws in one dimension, Comput. Math. Applic. 15 (1988), 595-602.

15. T. Johansen, A. Tveito, R. Winther, A Riemann solver for a two-phase multicomponent process, SIAM J. Sci. Stat. Comput. 10 (1989), 846-879.

16. R.J. LeVeque, A large time step shock-capturing technique for scalar conservation laws, SIAM J. Numer. Anal. 19 (1982), 1051-1073.

17. B.J. Lucier, A moving mesh numerical method for hyperbolic conservation laws, Math. Comp. 46 (1986), 59-69.

18. N.H. Risebro, A front tracking alternative to the Random choice method, Preprint Series, Institute of Mathematics, University of Oslo (1989).

19. J. Smoller, "Shock Waves and Reaction-Diffusion Equations," Springer, New York, 1983.

20. G.A. Sod, A numerical study of a converging cylindrical shock, J. Fluid Mech. 83 (1977), 785-794.

21. P. Woodward, Trade offs in designing explicit hydrodynamical schemes for vector computers, in "Parallel computations," Edited by G. Rodrigue, Academic Press, New York, 1982, pp. 153-171. 
22. P. Woodward, P. Colella, The numerical simulation of two dimensional fluid flow with strong shocks, J. Comput. Phys. 54 (1984), 115-173.

Keywords. Conservation laws, front tracking, Riemann problem

1980 Mathematics subject classifications.35L65, 65M99, 35R60 


\section{TABLE 1}

\begin{tabular}{rrccc}
$\delta$ & \multicolumn{4}{c}{ Front tracking } \\
50.0 & CPU & Error & Mass err\% & Energy err\% \\
40.0 & 10.8 & 0.165 & 0.71 & 0.92 \\
30.0 & 17.8 & 0.098 & 0.51 & 0.60 \\
25.0 & 26.4 & 0.069 & 0.38 & 0.47 \\
20.0 & 37.0 & 0.056 & 0.39 & 0.41 \\
15.0 & 86.7 & 0.037 & 0.25 & 0.29 \\
12.5 & 134.8 & 0.030 & 0.24 & 0.30 \\
10.0 & 193.7 & 0.026 & 0.19 & 0.25 \\
7.5 & 391.1 & 0.020 & 0.16 & 0.23 \\
7.0 & 399.9 & 0.018 & 0.14 & 0.20 \\
6.0 & 690.3 & 0.017 & 0.12 & 0.19 \\
5.0 & 810.3 & 0.014 & 0.09 & 0.14
\end{tabular}

Based on a standard regression analysis we compute:

$\boldsymbol{E}=\mathbf{O}\left(\delta^{1.02}\right) \quad$ Standard error estimate for the exponent: 0.03 CPU-time $=\mathbf{O}\left(\varepsilon^{-2.1}\right)$ Standard error estimate for the exponent: 0.06 


\section{TABLE 2}

\section{Front tracking}

\begin{tabular}{ccl}
$\delta$ & \multicolumn{1}{c}{ CPU } & error \\
1.0 & 10.18 & .118 \\
0.9 & 13.30 & .095 \\
0.7 & 21.90 & .058 \\
0.6 & 42.06 & .045 \\
0.5 & 69.02 & .043 \\
0.4 & 121.74 & .040 \\
0.3 & 267.00 & .031 \\
0.2 & 799.66 & .020
\end{tabular}

Based on a standard regression analysis we compute:

$\varepsilon=\mathbf{O}\left(\delta^{1.9} \quad\right.$ Standard error estimate for the exponent: 0.1 CPU-Lime $=\mathbf{O}\left(\varepsilon^{-2.5}\right)$ Standard error estimate for the exponent: 0.3

Number of grid blocks for Glimm's method and Lax-Friedrichs method is $14.0 / \Delta x$.

\section{Glimm's method}

\begin{tabular}{lrr}
$\Delta \mathrm{x}$ & \multicolumn{1}{c}{ CPU } & error \\
0.10 & 6.7 & 0.708 \\
0.09 & 8.0 & 0.616 \\
0.08 & 13.1 & 0.726 \\
0.07 & 23.5 & 0.512 \\
0.06 & 26.8 & 0.410 \\
0.05 & 43.5 & 0.592 \\
0.04 & 61.7 & 0.372 \\
0.03 & 96.9 & 0.197 \\
0.02 & 219.2 & 0.231 \\
0.01 & 696.9 & 0.144
\end{tabular}

Based on a standard regression analysis we compute:

$\varepsilon=\mathbf{O}\left(\Delta x^{0.7}\right) \quad$ Standard error estimate for the exponent: 0.1 CPU-time $=O\left(\varepsilon^{-2.4}\right)$ Standard error estimate for the exponent: 0.4 


\begin{tabular}{rr}
\multicolumn{1}{c}{ CPU } & error \\
2.22 & 0.398 \\
2.72 & 0.367 \\
3.48 & 0.332 \\
4.42 & 0.303 \\
6.10 & 0.268 \\
8.98 & 0.234 \\
13.54 & 0.200 \\
55.24 & 0.159 \\
233.24 & 0.115 \\
347.44 & 0.070 \\
622.48 & 0.061 \\
1403.46 & 0.031
\end{tabular}

Based on a standard regression analysis we compute: $\varepsilon=\mathbf{O}\left(\Delta x^{0.81}\right) \quad$ Standard error estimate for the exponent: 0.03 CPU-time $=\mathbf{O}\left(\varepsilon^{-2.4}\right)$ Standard error estimate for the exponent: 0.1 


\section{TABLE 3.}

Number of grid blocks for Lax-Friedrichs method is $65.0 / \Delta \mathrm{x}$.

\begin{tabular}{|c|c|c|}
\hline$\Delta \mathrm{x}$ & $\mathrm{CPU}$ & error \\
\hline 0.5 & 2.98 & .171 \\
\hline 0.4 & 4.34 & .145 \\
\hline 0.3 & 7.64 & .118 \\
\hline 0.2 & 17.02 & .087 \\
\hline 0.1 & 66.62 & .051 \\
\hline
\end{tabular}

Based on a standard regression analysis we compute: $\boldsymbol{\varepsilon}=\mathbf{O}\left(\Delta x^{0.75}\right) \quad$ Standard error estimate for the exponent: 0.01 CPU-time $=\mathbf{O}\left(\varepsilon^{-2.58}\right)$ Standard error estimate for the exponent: 0.02

\begin{tabular}{rrr}
\multicolumn{3}{c}{ Eront tracking } \\
$\delta$ & CPU & error \\
12.0 & 1.42 & 0.056 \\
10.0 & 1.86 & 0.049 \\
8.0 & 3.42 & 0.042 \\
6.0 & 6.02 & 0.034 \\
4.0 & 13.50 & 0.024 \\
3.0 & 24.26 & 0.022 \\
2.0 & 62.86 & 0.012
\end{tabular}

Based on a standard regression analysis we compute: $\boldsymbol{\varepsilon}=\mathbf{O}\left(\delta^{0.8}\right) \quad$ Standard error estimate for the exponent: 0.1 CPU-time $=\mathbf{O}\left(\varepsilon^{-2.5}\right)$ Standard error estimate for the exponent: 0.1 


\section{Figure captions.}

Fig 2.1 The approximation of a rarefaction wave in state space.

Fig 2.2 The approximation of a rarefaction wave in $x-t$ space.

Fig 2.3 A system of discontinuities in $x$ - $t$ space.

Fig 3.1 The curves $u_{1}$ and $u_{2}$ in $u-p$ space.

Fig 4.1 Problem \#1. All fronts from $t=0$ to $t=0.038$ for the approximation using $\delta=100.0$.

Fig 4.2 Problem \#1. The reference solution and the approximation with $\delta=20.0$ at $t=0.038$.

Fig 4.3 Problem \#2. The initial density distribution.

Fig 4.4a Problem \#2.The reference solution and front tracking, $\delta=1.0$. CPU-time $=10.1$.

Fig 4.4b Problem \#2. The reference solution and Glimm's method, $\Delta x=0.02$. CPU-time $=219.2$.

Fig 4.4c Problem \#2. The reference solution and Lax-Friedrichs method, $\Delta x=0.04$. CPU-time $=13.5$.

Fig 4.5 Problem \#3. The initial density distribution.

Fig 4.6a Problem \#3. The reference solution and front tracking, $\delta=12.0$. CPU-time $=1.4$.

Fig 4.6b Problem \#3. The reference solution and Lax-Friedrichs method, $\Delta x=0.2$. CPU-time $=17.0$. 


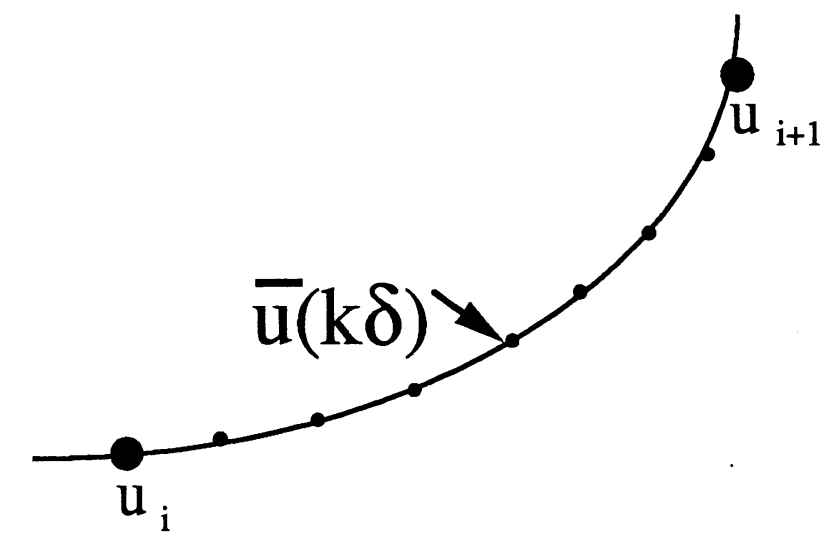

figure 2.1

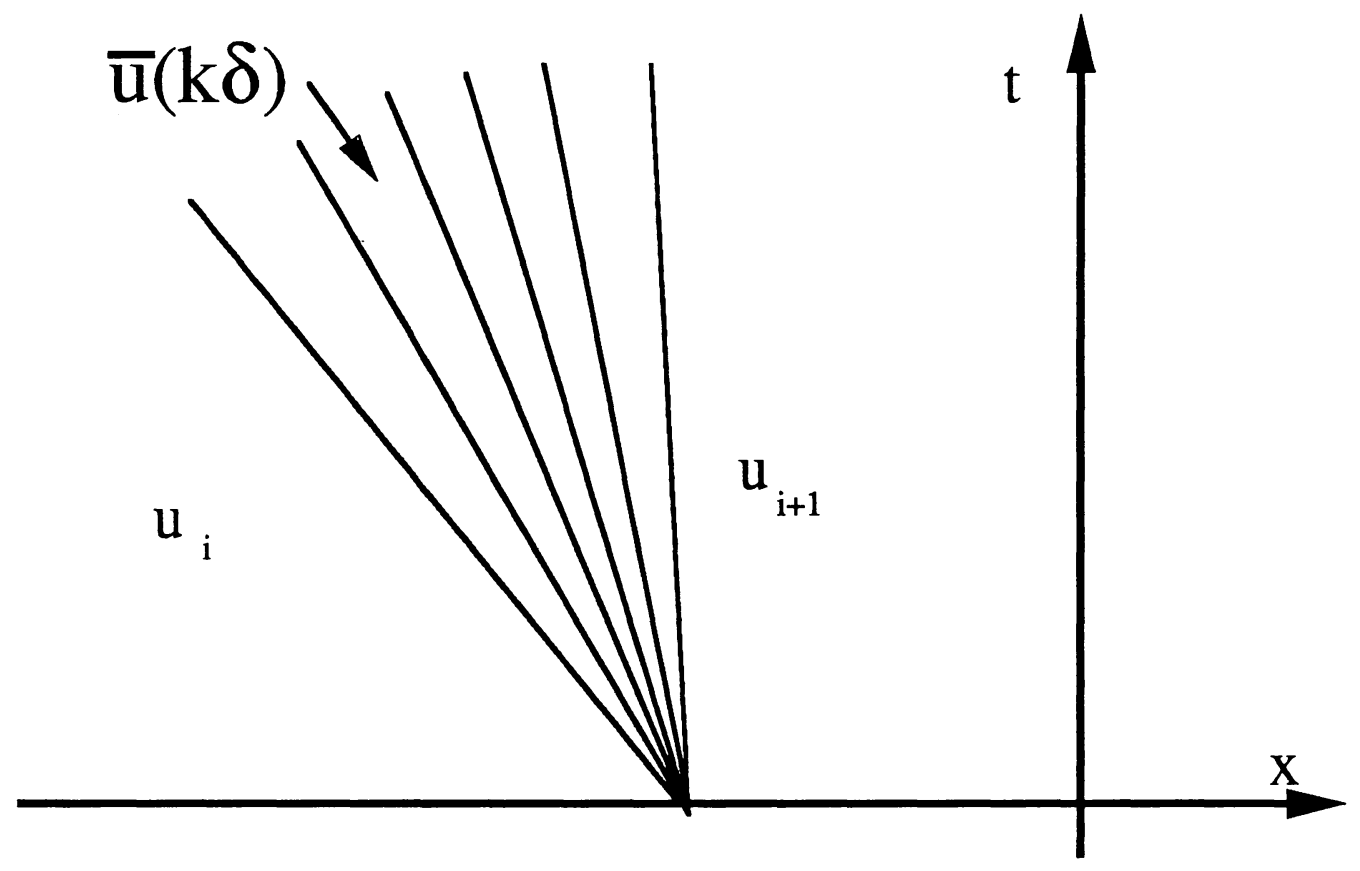

figure 2.2 


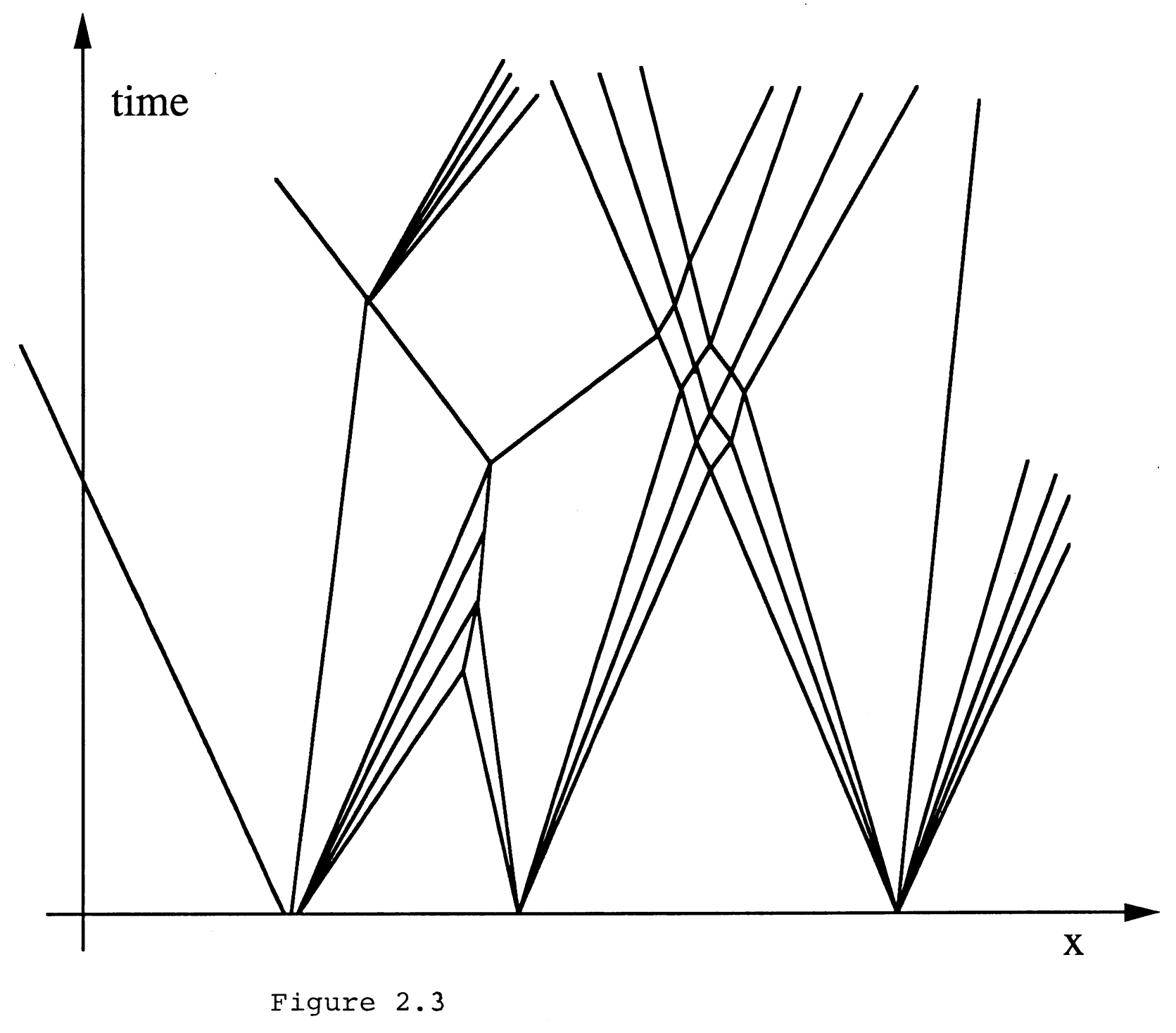




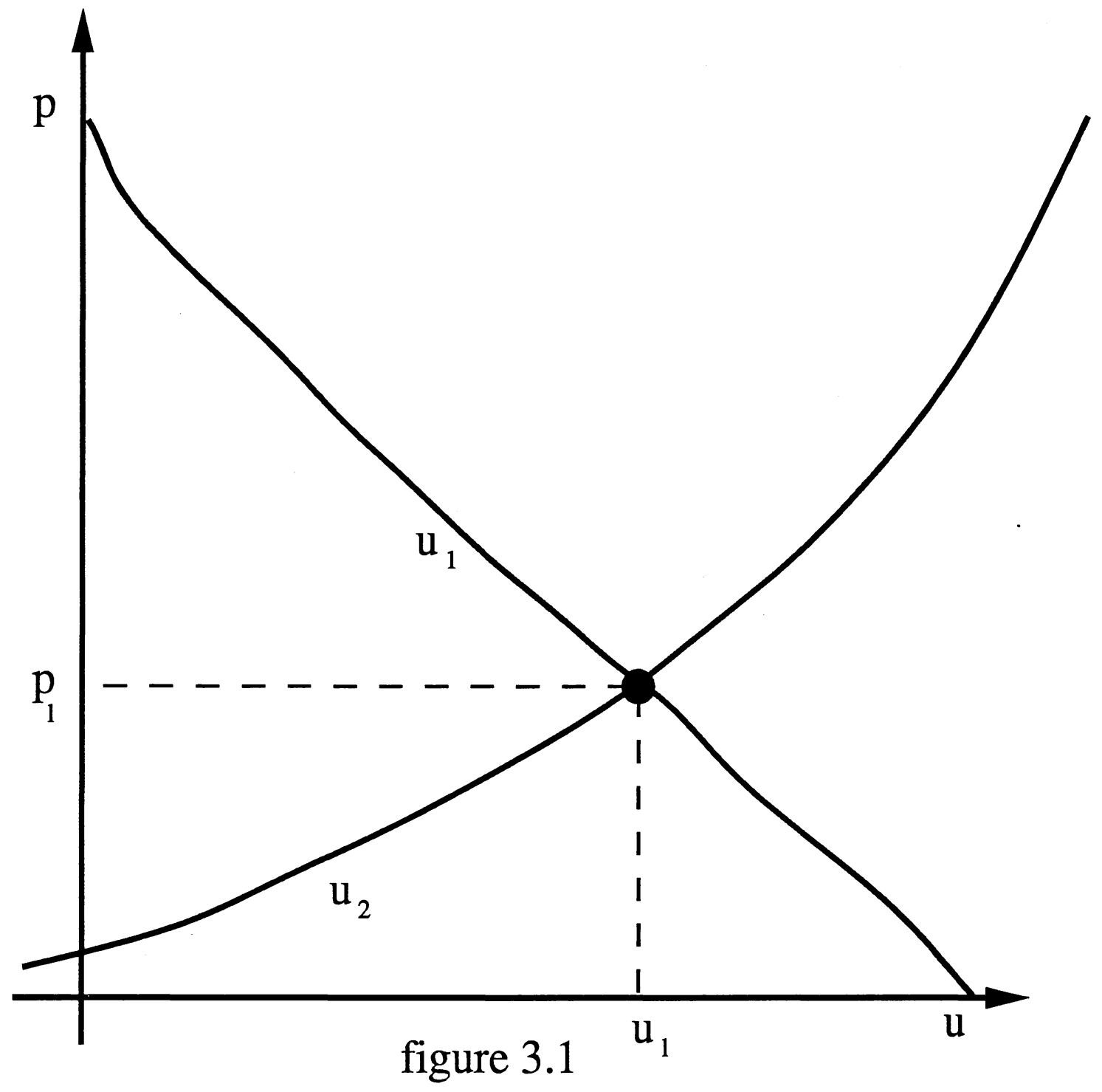




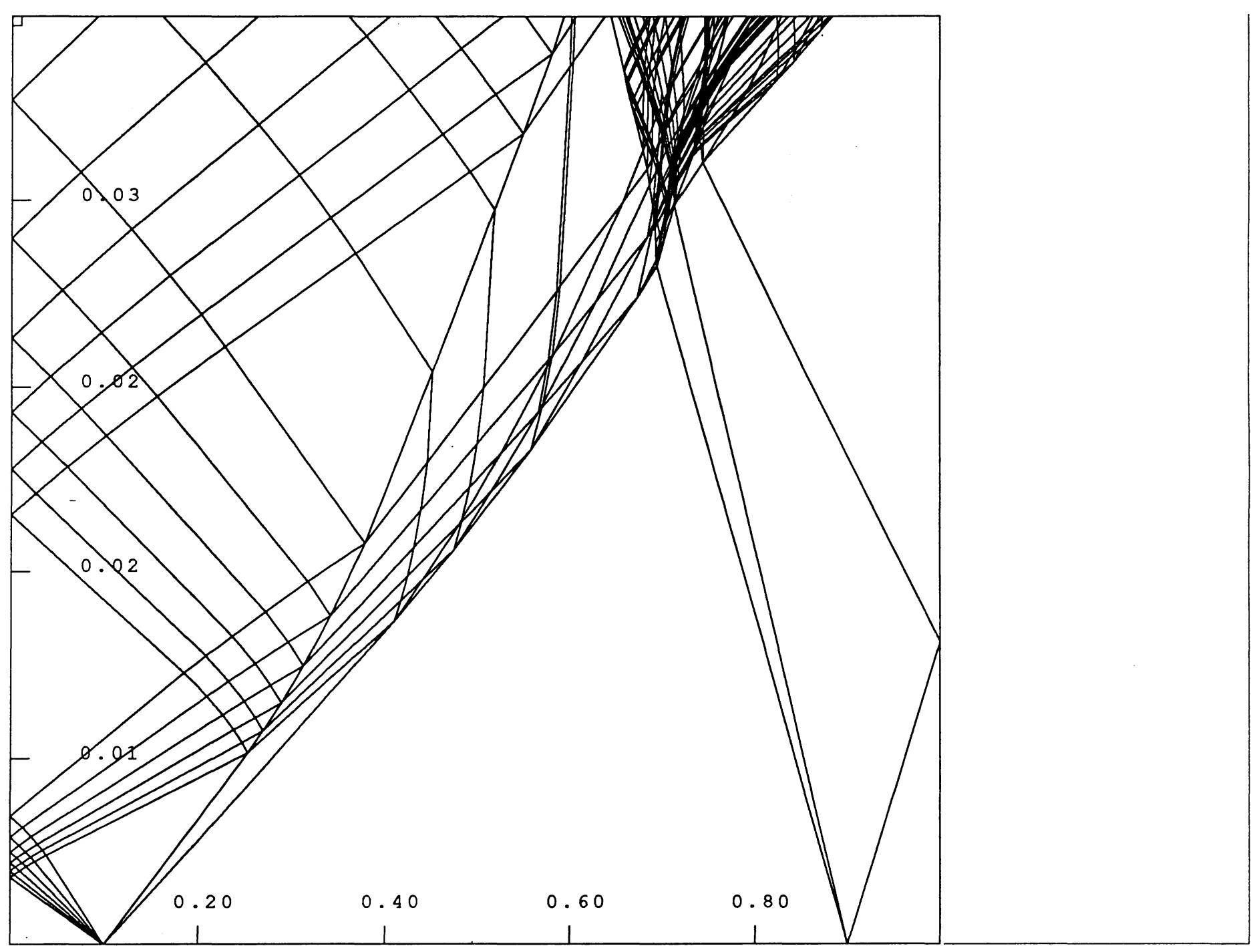

Figure 4.1 


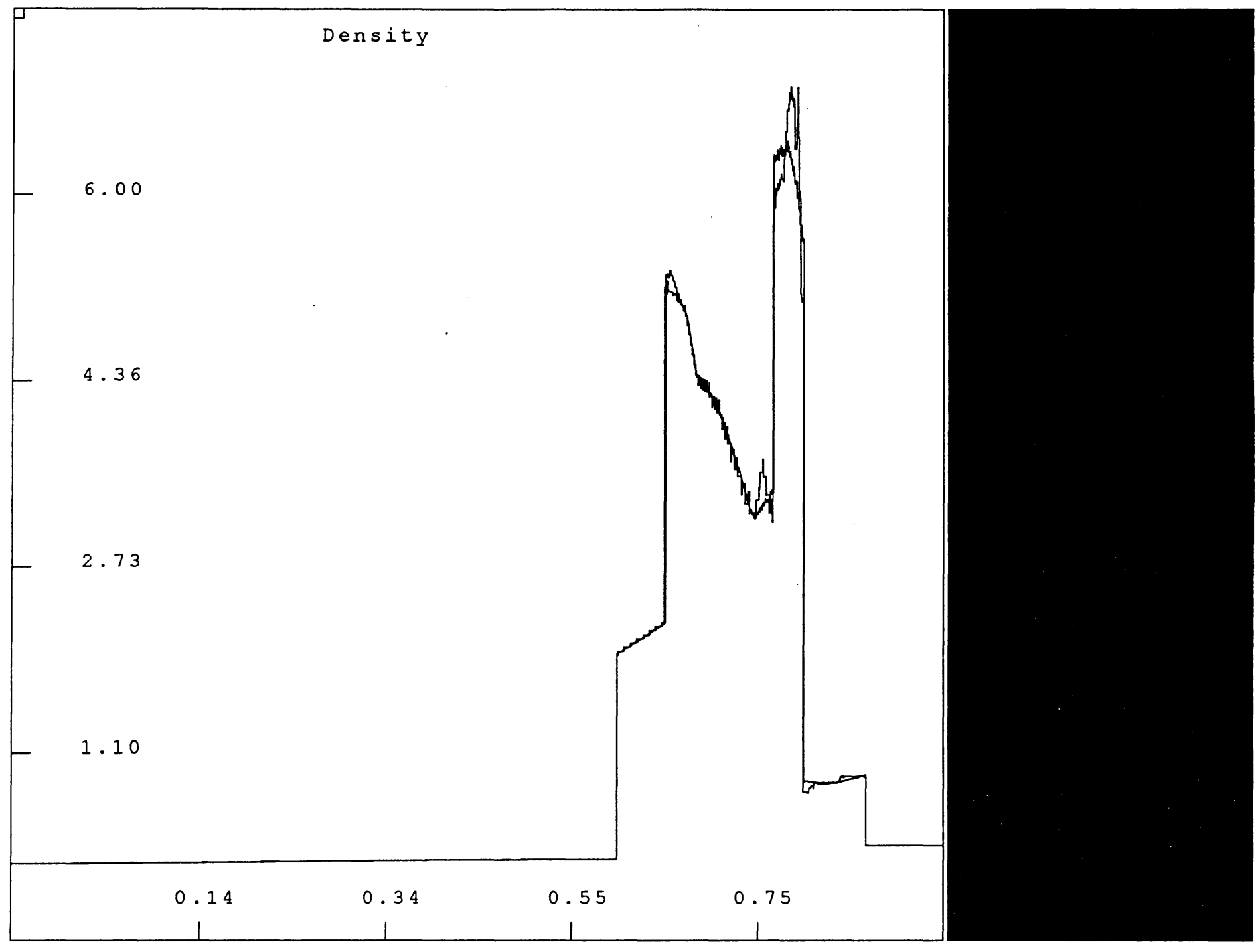

Figure 4.2 


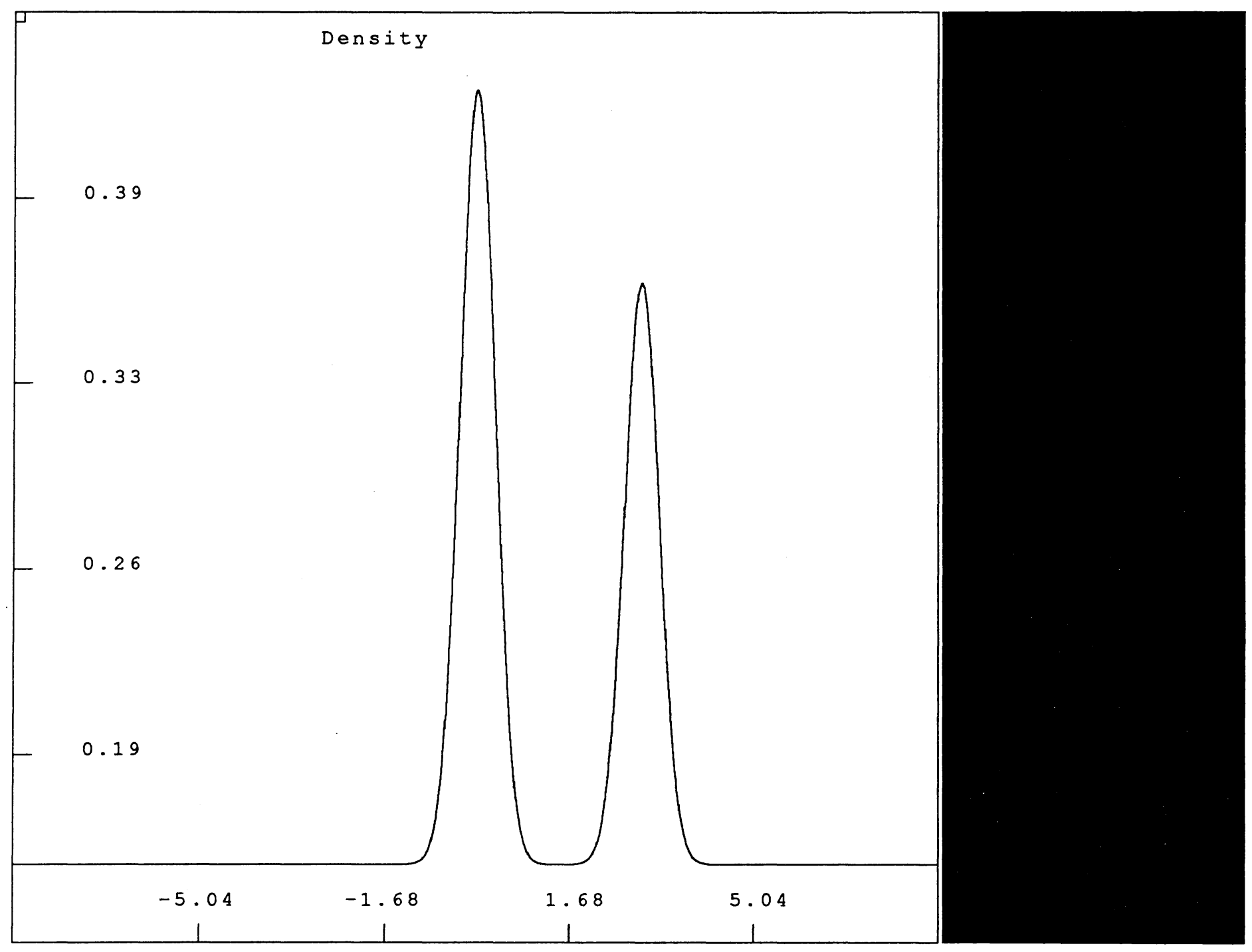

Figure 4.3 


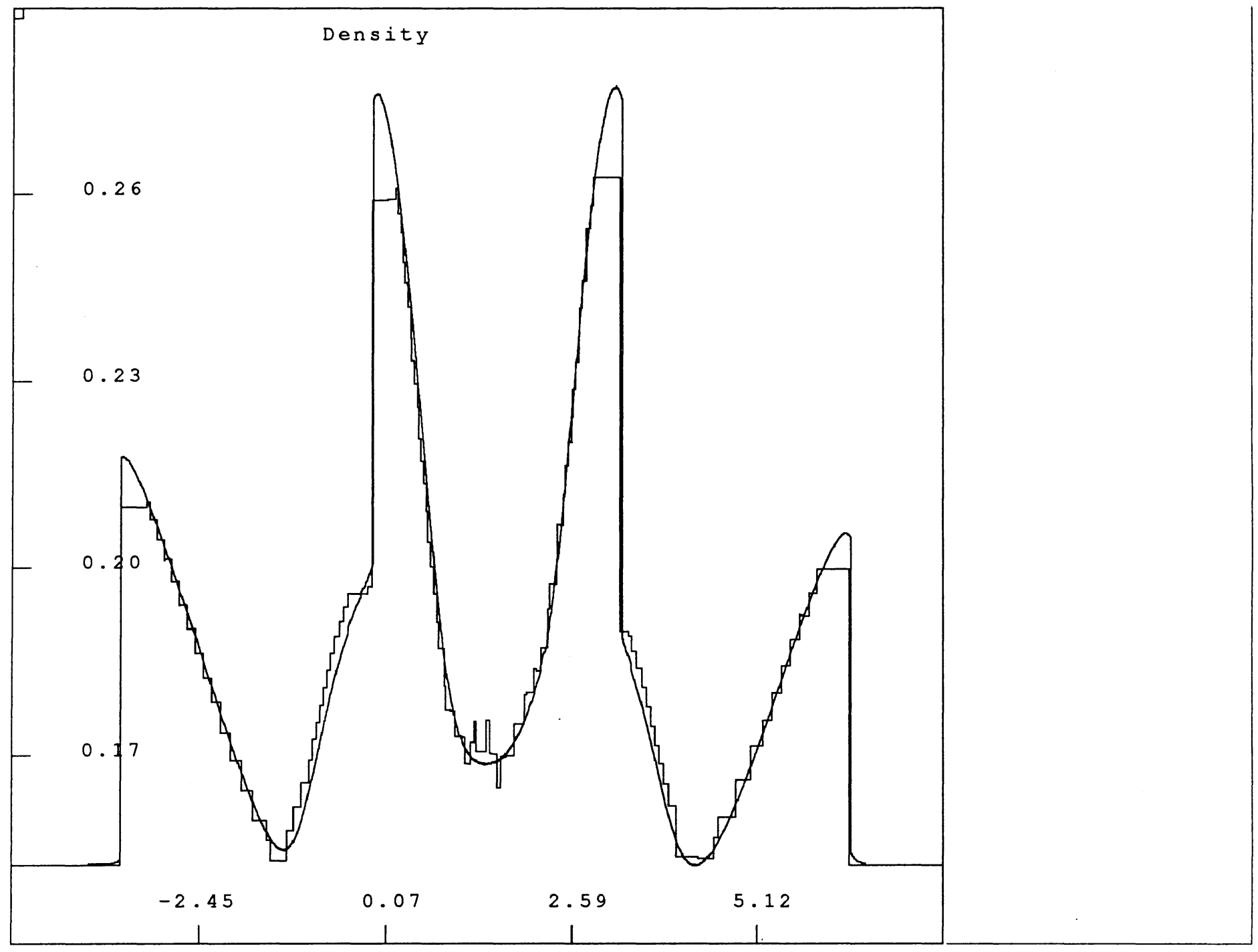

Figure $4.4 \mathrm{a}$ 


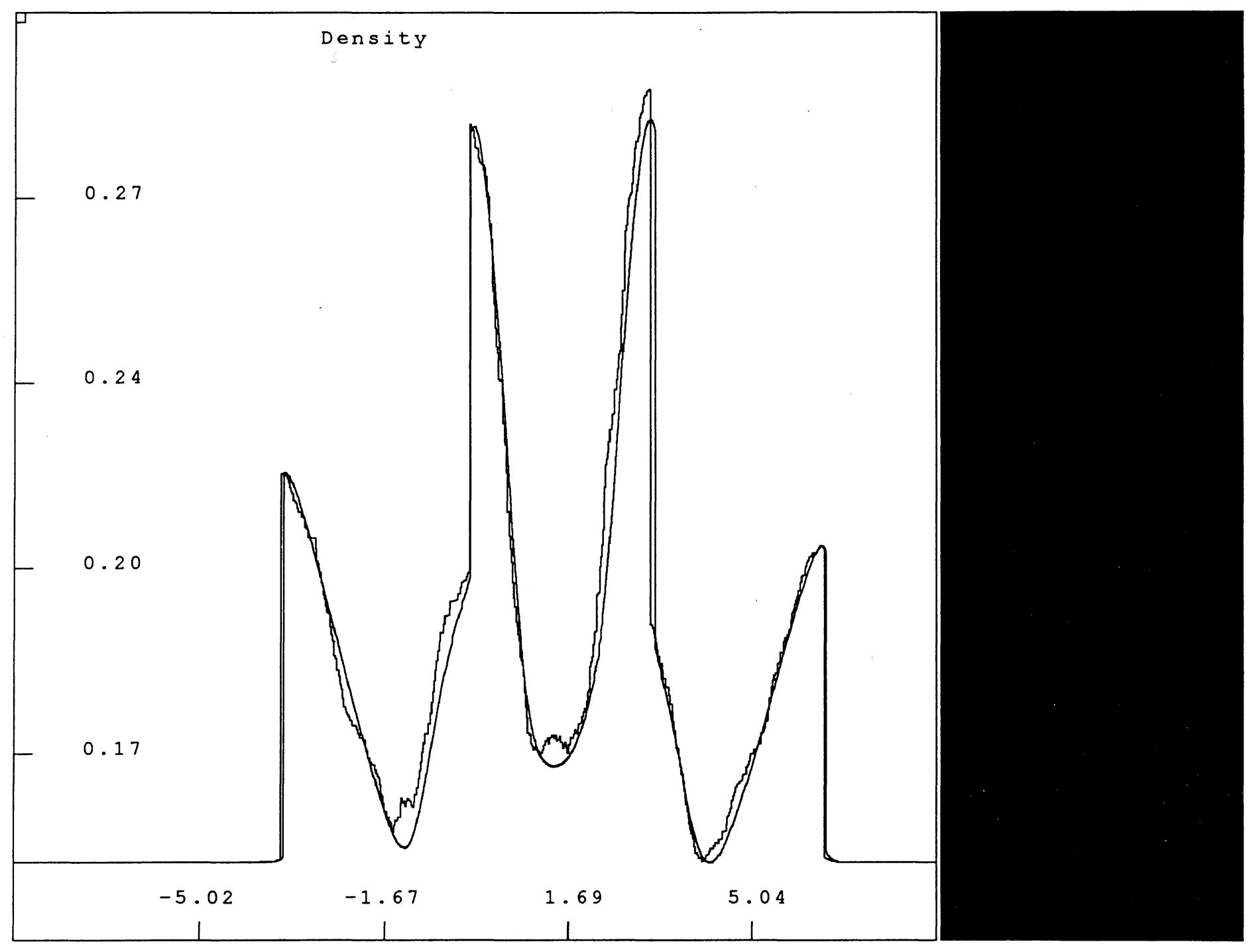

Figure $4.4 \mathrm{~b}$ 


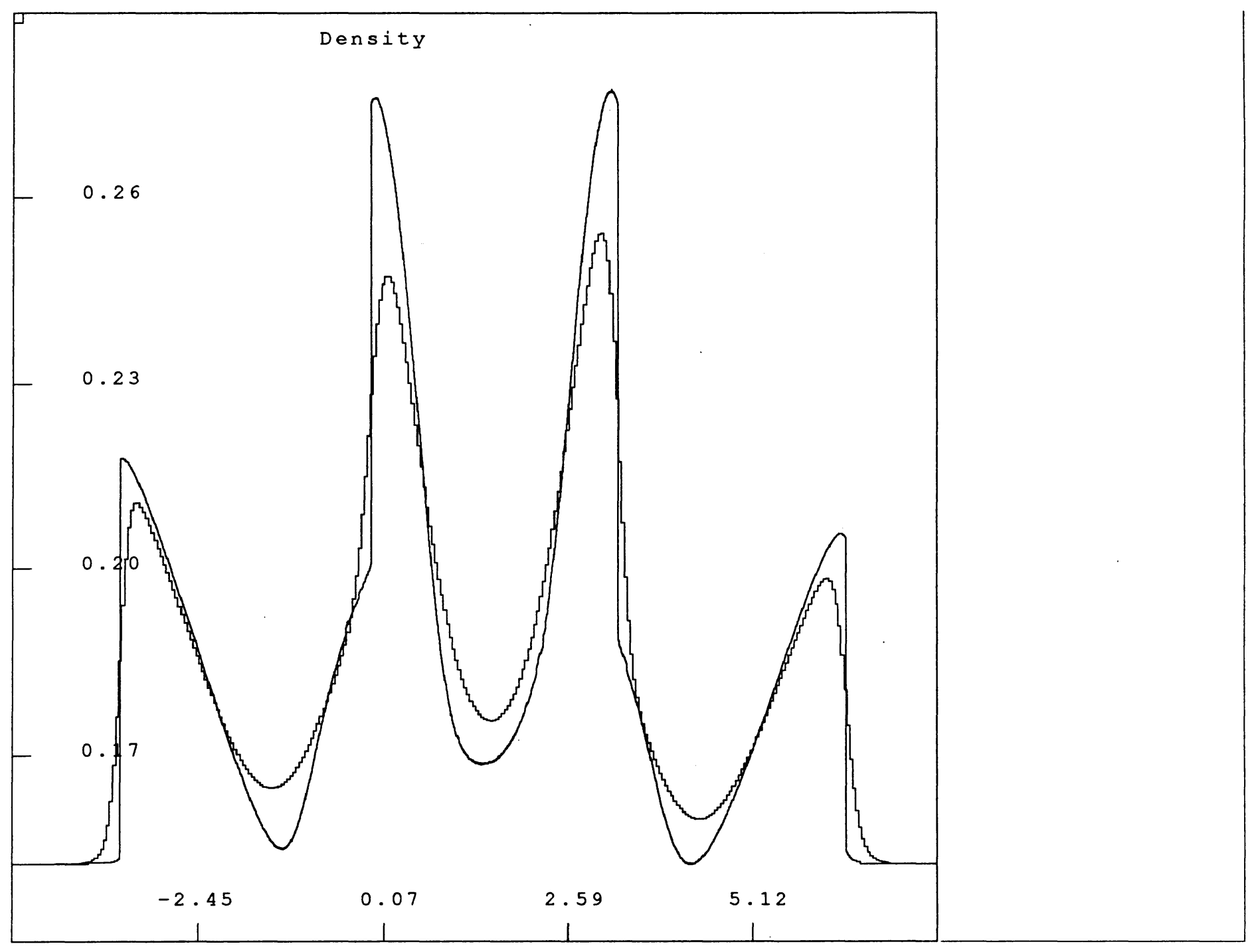

Figure $4.4 \mathrm{C}$ 


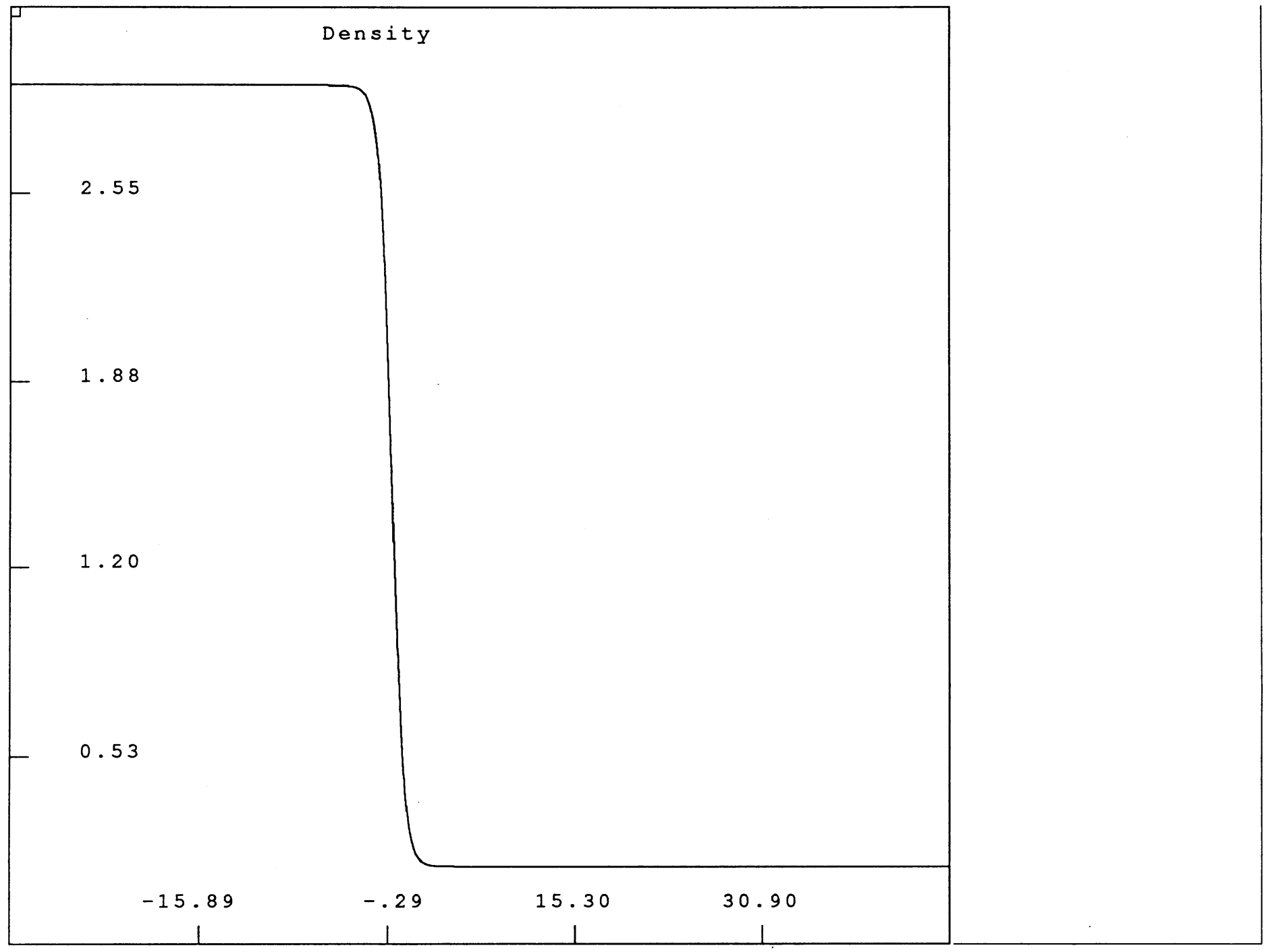

Figure 4.5 


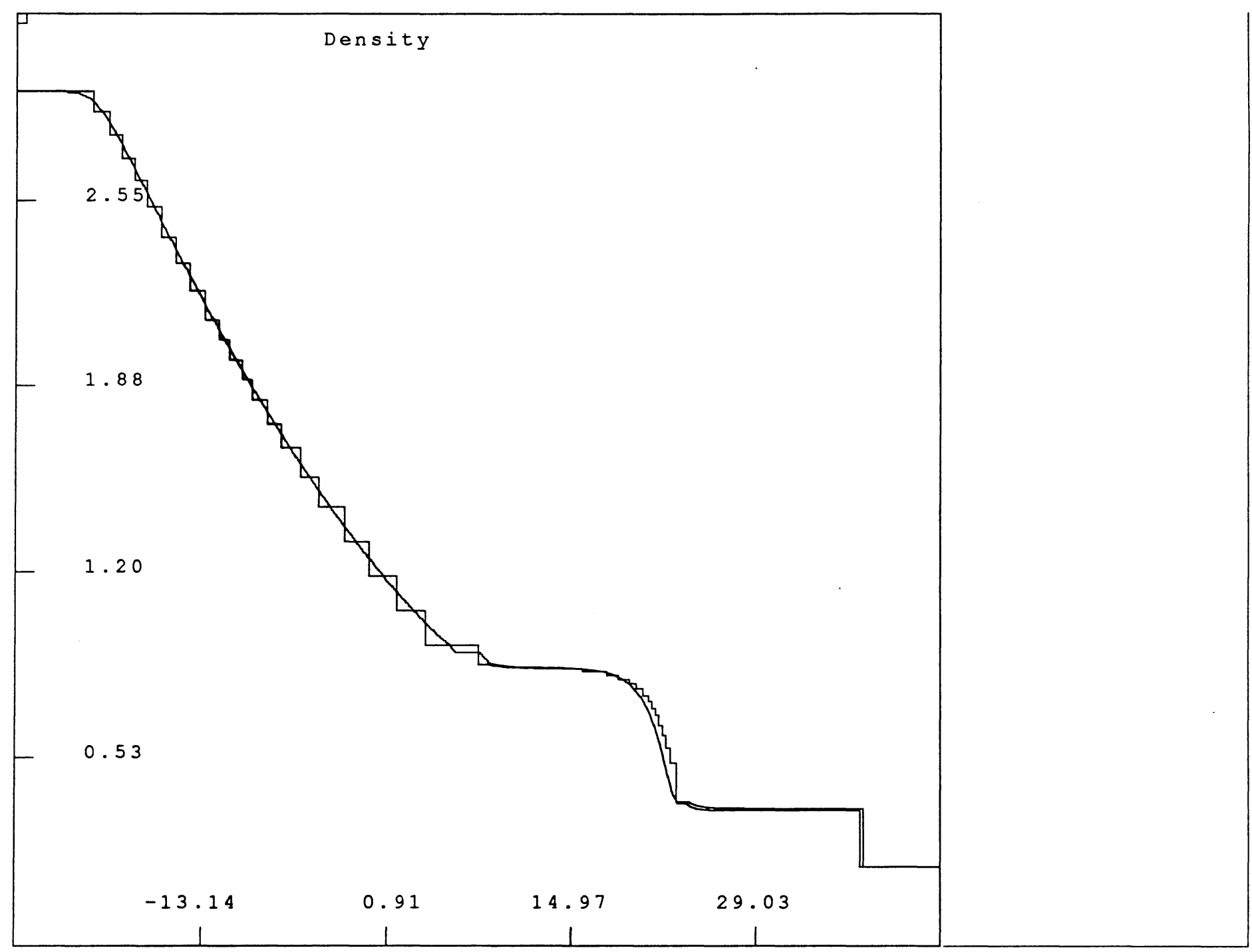

Figure $4.6 a$ 


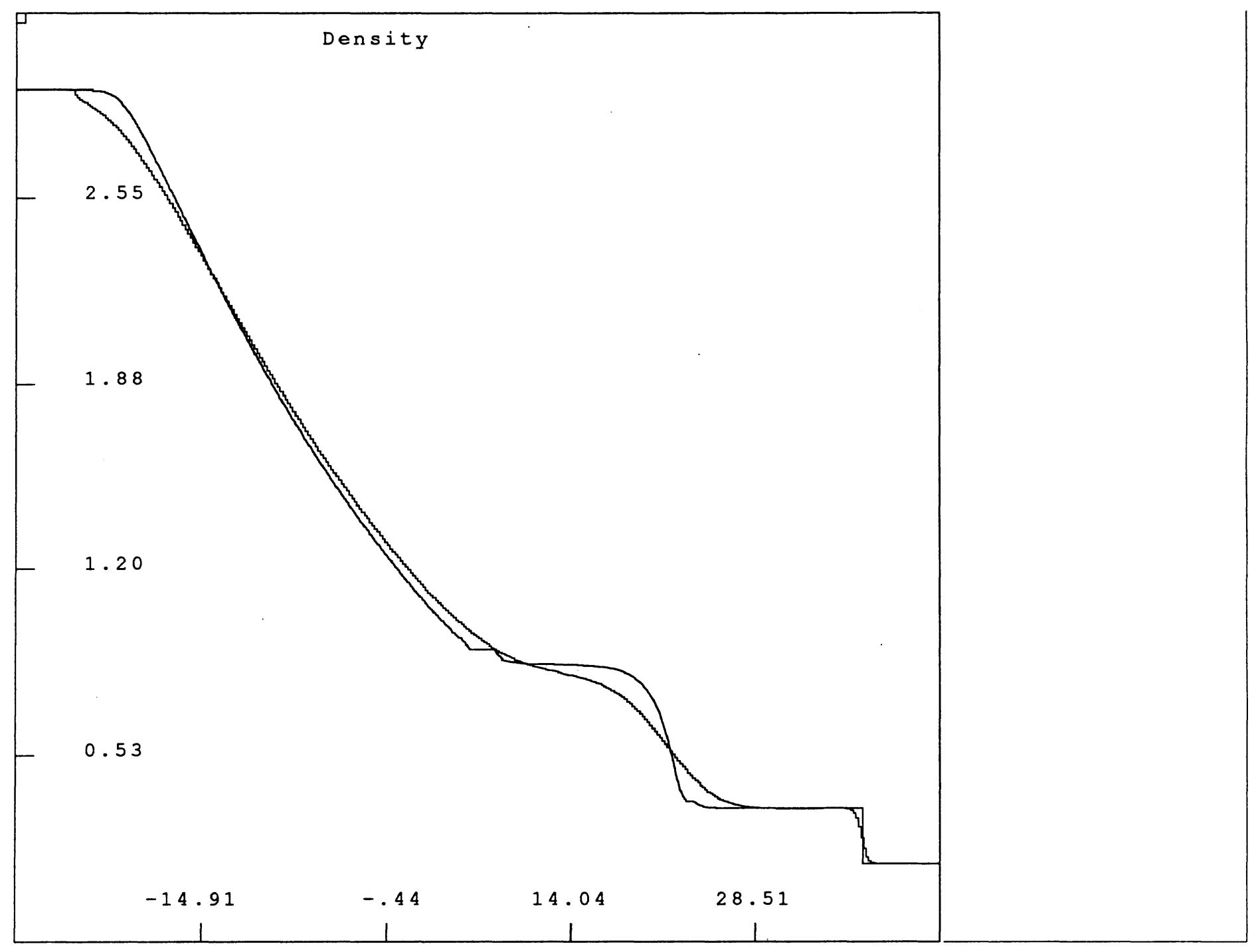

Figure $4.6 b$ 